\title{
On the relationship between surface tension and viscosity of fluids
}

Hadi Ahmari ${ }^{\text {, }}$ Mohammad Chalkesh Amiri ${ }^{2}$

${ }^{I}$ Department of Chemical Engineering, Quchan Branch, Islamic Azad University, Quchan, Iran. ${ }^{2}$ Department of Chemical Engineering, Isfahan University of Technology, Isfahan, 84156-83111, Iran. Article Info: Submitted on October 24, 2014, Accepted on July 15, 2015.

\begin{abstract}
Relating surface tension and viscosity of liquids not only is important conceptually but it can also be used to test the validity of some measured data. Schonhorn modified Pelofsky's equation by introducing the concept of the vapor viscosity $\eta_{\vee}$ in equilibrium with the liquid, i.e. $\gamma=A \exp \left(-B /\left(\eta_{l}-\eta_{v}\right)\right)$. This empirical relation can be applied to both organic and inorganic solutions of pure and mixed components. As surface tension is a strong function of surfactant, they neglected to emphasis that mixed components must not be surface active agents. Both Pelofsky and Schonhorn indicated that A may be an indication of the surface tension of the liquid at the temperature where $\eta=\infty$. They were not successful in correlating the temperature where $\eta \rightarrow \infty$ and the surface tension at the melting point. In this work, a new empirical equation for relating surface tension and viscosity has been developed, i.e., $\gamma\left(\left(\mathrm{T}_{\mathrm{c}}-\mathrm{T}_{\mathrm{m}}\right) /\left(\mathrm{T}_{\mathrm{c}}-\mathrm{T}\right)\right)=\delta(1-\beta / \eta)$. This equation not only satisfies the extreme conditions but it also reveals a physical concept for $\delta$, that is, it should equal the surface tension at the melting point of the liquid.
\end{abstract}

Keywords: Surface tension; Viscosity; Pelofsky; Physical concept; Schonhorn.

\section{Introduction}

Surface tension not only determines the quality of many of the products resulting from different industries but also affects the efficiency of processes such as distillation, extraction and detergency.

The literature information for surface tension is considerably smaller than that available for viscosity. The results of surface tension measurement are very sensitive to the presence of small amounts of impurities. ${ }^{2}$ The reported values are usually scattered even in standard textbooks. For example, Adamson ${ }^{1}$ mentioned a surface tension of $22.4 \mathrm{mN} / \mathrm{m}$ for Ethanol from Jasper $^{3}$ in a table but mentioned $22.75 \mathrm{mN} / \mathrm{m}$ from international critical table (ICT) in another table. Novakovic et al. ${ }^{4}$ presented a comparison between surface tension data of gold from the Au-melting point to $1573 \mathrm{~K}$, reported by different authors. They showed that range of reported values of the surface tension of gold at the melting point is from 1105 to $1182 \mathrm{mN} / \mathrm{m}$, that is, a difference of $77 \mathrm{mN} / \mathrm{m}$.

Viscosity, a measure of liquid resistance to flow, is a key parameter in fluid's behavior. Several product characteristics depend on the magnitude of viscosity. As a liquid is cooled its viscosity normally increases, but viscosity also has a tendency to prevent crystallization. When a liquid is cooled to below its melting point, crystals usually form and it solidifies; but sometimes it can become super-cooled and remain liquid below its melting point because lack of nucleation sites to initiate the crystallization. Solids at temperatures in the vicinity of the melting point, the same temperature range in which plasticity is found, contain a significant proportion of liquid molecules. The liquid molecule has zero fluidity (reciprocal viscosity) below the liquid temperature, which corresponds to the melting point of the solid.

Although experimental measurement of viscosity and surface tension is not difficult, the measured values of surface tension are highly exposed to errors due to environmental conditions. ${ }^{5}$ Therefore, relating surface tension and viscosity is not only important conceptually but can also be used to test the validity of measured data.

Pelofsky ${ }^{6}$ proposed the following surface tensionviscosity relationship for a large variety of liquids:

$\gamma=A \exp (-B / \eta)$

where $\mathrm{A}$ and $\mathrm{B}$ are constants. He found that this empirical relation can be applied to both organic and inorganic solutions of pure and mixed components. Considering the surface tension as a strong function of surfactant, the mixed components must be non surface active agents even though Pelofsky and others surprisingly neglected this important point.

Aromatics such as, benzene, toluene, xylene and phenol, n-alkanes in the range $\mathrm{n}, \mathrm{n}$-alcohols in the range $\mathrm{CH}_{3} \mathrm{OH}-\mathrm{C}_{4} \mathrm{H}_{9} \mathrm{OH}$, also water and some aqueous solutions were shown to follow the above relation. 


\section{Chemical Engineering Research Bulletin 18(2015) 18-22}

Later, Schonhorn ${ }^{7}$ found that eqn. 1 can apply only to a restricted temperature range and necessarily fails at the critical temperature as viscosity is finite while surface tension is zero. He correctly extended eqn. 1 by introducing the concept of the vapor viscosity $\eta_{v}$ in equilibrium with the liquid. He proposed the following equation:

$$
\gamma=A \exp \left(-B /\left(\eta_{l}-\eta_{v}\right)\right)
$$

where $\eta_{1}$ and $\eta_{v}$ are the material's liquid and vapor viscosity respectively. This form of relation between surface tension and viscosity satisfies the extreme condition that at critical temperature surface tension must be zero. When $\left.\left.\eta_{l}\right\rangle\right\rangle \eta_{V}$, which is at $T\left\langle\left\langle T_{c}\right.\right.$, eqn. 1 is obtained. Schonhorn's modification of eqn. 1 was excellent not only for satisfying the extreme condition but also for including the experimental fact that the surface tension of each liquid must be measured in equilibrium with its vapor. Eqn. 2 was successfully applied to various liquids such as Sodium, potassium, n-Decane, Argon, Benzene, water, and salts such as $\mathrm{NaBr}$ and $\mathrm{NaCl}$.

Both Pelofsky and Schonhorn indicated that A may be an indication of the surface tension of the liquid at the temperature where $\eta=\infty$. They attempted to correlate between the temperature where $\eta \rightarrow \infty$ and the temperature of homogeneous nucleation. Homogeneous nucleation as opposed to heterogeneous nucleation results from a spontaneous phase change without the assistance of impurities. The principal component of the energy barrier in homogeneous nucleation is the thermal energy that keeps particles from clustering. Opposing these forces are the intermolecular attractive forces. When attractive forces exceed the thermal and surface forces, irreversible formation of a new phase occurs.

Recently, Queimada et al. ${ }^{8}$ have checked the validity of eqn. 2 for several pure and mixed n-alkanes. In fact, they used eqn. 1 since they found most of the available data has been taken far from the critical point, where the vapor phase viscosity is small compared to that of the liquid phase. They verified eqn. 1 for some of the studied n-alkanes, $n-\mathrm{C}_{6} \mathrm{H}_{14}$ up to $\mathrm{n}-\mathrm{C}_{20} \mathrm{H}_{42}$. In their illustration, only two points in 163 happened to be outside the relation but these were also found to be outlinear in the surface tension or viscosity plots as a function of temperature. They also checked and applied this simple equation to real fluids; three petroleum distillation cuts of different origins were selected for evaluation. Plots of $\ln \gamma$ as a function of $1 / \eta$ confirmed that eqn. 1 continues to be valid, even for multicomponent fluids containing components from different families, as already demonstrated in a previous work by Pedersen et al. ${ }^{9}$ They concluded that if a set of values of $\gamma$ and $\eta$ do not follow eqn. 1 that may indicate incorrect viscosity or surface tension measurements.

In his work, Schonhorn related A to the surface tension at $\mathrm{T}_{\mathrm{N}}$, the temperature of homogeneous nucleation, that is, the temperature when clusters start to form spontaneously. At this temperature the viscosity tends to infinite and thus $\gamma=\mathrm{A}$.

It should be noted that $\mathrm{T}_{\mathrm{N}}$ represents a temperature below which there is no liquid phase but only a crystalline or glassy state. To find $\mathrm{T}_{\mathrm{N}}$, the surface tension data for a liquid should be plotted as a function of temperature and extrapolated to lower temperature by assuming $\frac{\partial \gamma}{\partial T}$ to be constant. In general, the slope of $\gamma$ versus $\mathrm{T}$ is sufficiently linear to warrant such an approximation. ${ }^{1}$ The temperature at which $\gamma=\gamma_{N}$ is defined as $\mathrm{T}_{\mathrm{N}}$. Although the values of for a variety of liquids are reported, the results show that $T_{N}$ has no physical concept.

Schonhorn and others were not successful in interpreting $\mathrm{A}$; therefore, developing a better correlation between surface tension and viscosity to reveal a physical concept for $\mathrm{A}$ is desirable. This work is going to satisfy this need.

\section{Materials and Methods}

The plot of $\ln \gamma$ as a function of fluidity, $1 / \eta$, is linear. Investigating these plots for several liquids reveals that when viscosity is high the (negative) slope (equal to B) is also high and vice versa. As a result it seems that a change in the value of $(B / \eta)$ should not be high. This result was confirmed with some liquids, pure n-alkanes and also n-alkane mixtures. It was found that $\mathrm{B}$ is a function of average molecular weight as viscosity is. This finding is also checked with literature information ${ }^{8,10}$ as shown in Table 1 . Viscosities of these petroleum distillation cuts are measured not calculated values. The authors attempted to predict viscosities and surface tensions of fuel (petroleum distillation cuts) by a new corresponding states model by using their data.

We also calculated $(B / \eta)$ for petroleum distillation cuts at various temperatures as shown in Table 2 . It should be noted that fit lines describe the available data (fitted data) to within their experimental uncertainty bands.

The most interesting result of Tables 1 and 2 is that even for mixtures the value of $(B / \eta)$ is much smaller than 1 at normal operating temperature. These findings encouraged us to propose the following equation to relate surface tension and viscosity at any temperature, $\mathrm{T}$, in absence of surface active agents:

$$
\gamma=\delta \frac{T_{c}-T}{T_{c}-T_{m}}\left(1-\frac{\beta}{\eta}\right)
$$




\section{Chemical Engineering Research Bulletin 18(2015) 18-22}

Here $\delta$ and $\beta$ are constant; $\mathrm{T}_{\mathrm{c}}$ and $\mathrm{T}_{\mathrm{m}}$ are critical and melting temperatures respectively. This equation can be written as

$\gamma \frac{T_{c}-T_{m}}{T_{c}-T}=\delta-\frac{\delta \beta}{\eta}$

If this equation is going to be valid, then it is necessary to fulfill the following two conditions:

(i) it must satisfy extreme conditions,

(ii) a plot of $\gamma\left(\left(T_{c}-T_{m}\right) /\left(T_{c}-T\right)\right)$ as a function of $1 / \eta$ must be linear

Table 1: $(B / \eta)$ for petroleum distillation cuts at $293.2 \mathrm{~K}$.

\begin{tabular}{|c|c|c|c|c|}
\hline $\begin{array}{c}\text { Distillation } \\
\text { Cuts }\end{array}$ & MW & $\begin{array}{c}-\mathrm{B} \\
(\mathrm{mPa} . \mathrm{s})\end{array}$ & $\begin{array}{c}\eta \\
(\mathrm{mPa} . \mathrm{s})\end{array}$ & $-\mathrm{B} / \eta$ \\
\hline Troll & 231.7 & 0.438 & 6.45 & 0.0679 \\
\hline Brent & 222.8 & 0.408 & 5.96 & 0.0684 \\
\hline Sahara & 235.8 & 0.404 & 5.81 & 0.0695 \\
\hline
\end{tabular}

Table 2: $(B / \eta)$ for petroleum distillation cuts at various temperatures

\begin{tabular}{|c|c|c|c|c|c|c|}
\hline $\begin{array}{l}\text { T } \\
(\mathrm{K})\end{array}$ & 293.2 & 303.2 & 313.2 & 323.2 & 333.2 & 343.2 \\
Cuts & & & & & & \\
\hline Troll & 0.0679 & 0.093 & 0.121 & 0.154 & 0.190 & 0.227 \\
\hline Brent & 0.0684 & 0.098 & 0.126 & 0.157 & 0.192 & 0.231 \\
\hline Sahara & 0.0695 & 0.100 & 0.119 & 0.149 & 0.194 & 0.232 \\
\hline
\end{tabular}

\section{Results and Discussion}

Two extreme conditions can be considered for eqn. 3 . They are:

(i) at critical temperature, $T_{c}$, viscosity value is not zero but surface tension must be zero.

(ii) at melting point, where $\eta \rightarrow \infty$, surface tension must be a finite value.

It is easy to show that eqn. 3 satisfies both extreme conditions but in a different way from Pelofsky's equation. Plot of $\gamma\left(\left(T_{c}-T_{m}\right) /\left(T_{c}-T\right)\right)$ as a function of $1 / \eta$ for many liquids confirms that it is linear, as shown in Figs. 1 and 2 for some liquids. This data was compiled from various primary references. ${ }^{1,2,7,8,10}$ The slope of each line is equal to $\delta \beta$ and the intercept is $\delta$. For those liquids, plot of $\ln \gamma$ as a function of fluidity, $1 / \eta$, is also linear, therefore, both eqn. 1 and the developed equation in this work, eqn. 3, can well describe the relationship between surface tension and viscosity.

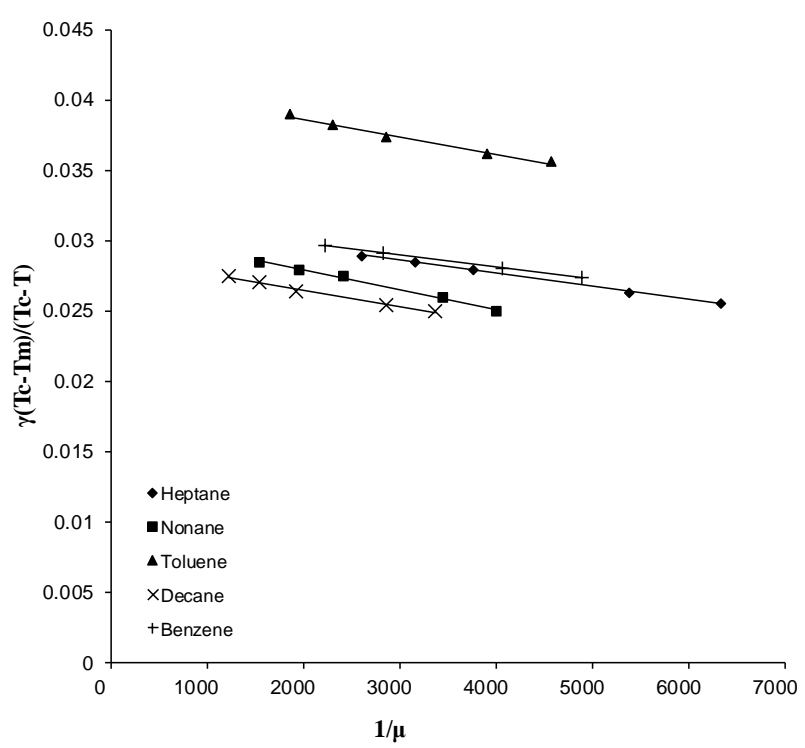

Figure 1: Plots of $\gamma\left(\left(T_{c}-T_{m}\right) /\left(T_{c}-T\right)\right)$ as a function of $1 / \eta$ for Heptane $(\bullet)$, Nonane $(\mathbf{\square})$, Toluene $(\boldsymbol{\Delta})$, Decane $(x)$ and Benzene $(+)$.

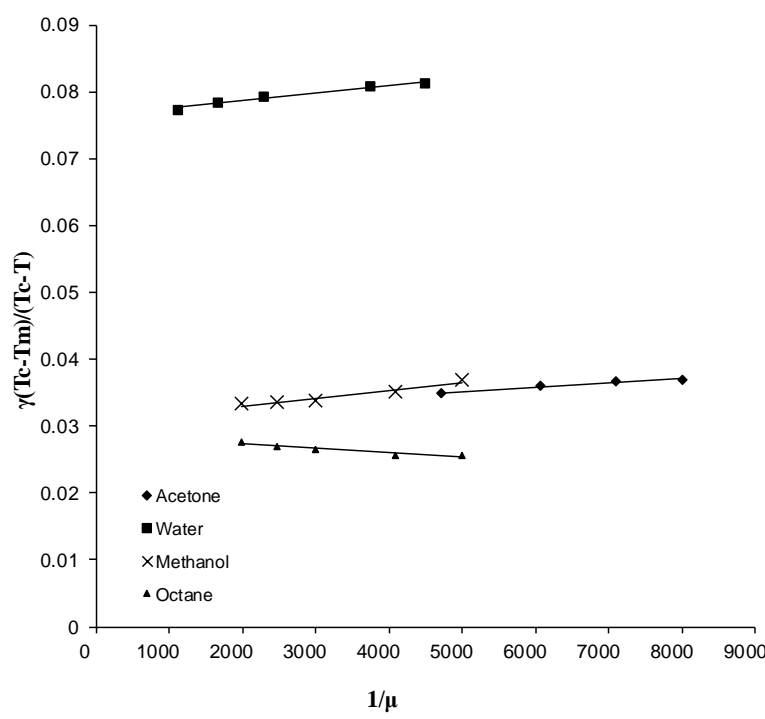

Figure 2: Plots of $\gamma\left(\left(T_{c}-T_{m}\right) /\left(T_{c}-T\right)\right)$ as a function of $1 / \eta$ for Acetone $(\star)$, Water $(\boldsymbol{\bullet})$, Methanol $(\times)$ and Octane ( $\boldsymbol{\Delta})$.

Comparison data between these two equations are shown in Table 3. For each liquid, A, $\delta, \mathrm{R}^{2}$ Pelofsky (R-squared value for Pelofsky's equation) and $\mathrm{R}^{2}$ Present (R-squared value for present work) were recorded in Table 3.

The results show that both equations correspond well with experimental data as R-squared values for both equations are usually more than 0.99 for most liquids. 


\section{Chemical Engineering Research Bulletin 18(2015) 18-22}

Table 3: Comparison data between present work, eqn.3, and others.

\begin{tabular}{|c|c|c|c|c|c|c|c|c|c|}
\hline Substance & $\begin{array}{c}\gamma \\
\left(\mathrm{at} 20^{\circ} \mathrm{C}\right)\end{array}$ & $\mathrm{d} \gamma / \mathrm{dT}$ & $\begin{array}{c}\mathrm{T}_{\mathrm{m}} \\
(\mathrm{K})\end{array}$ & $\begin{array}{c}\mathrm{T}_{\mathrm{c}} \\
(\mathrm{K})\end{array}$ & $\mathrm{A}$ & $\delta$ & $\gamma_{\mathrm{m}}$ & $\mathrm{R}_{\text {Pelofsky }}^{2}$ & $\mathrm{R}_{\text {Present }}^{2}$ \\
\hline Water & 72.9 & -0.2 & 273 & 647 & 79.0 & 76.4 & 76.9 & 0.9993 & 0.9997 \\
\hline Acetone & 24.0 & -0.11 & 178.4 & 508.2 & 38.8 & 31.5 & 35.96 & 0.9976 & 0.9898 \\
\hline Benzene & 28.9 & -0.13 & 278.5 & 562.2 & 38.0 & 31.5 & 30.79 & 0.9997 & 0.9963 \\
\hline Ethanol & 22.3 & -0.10 & 161 & 516.3 & 25.7 & 36.1 & 35.50 & 0.9986 & 0.988 \\
\hline Decane & 23.8 & -0.09 & 243.3 & 617.5 & 30.2 & 28.9 & 28.27 & 0.9988 & 0.9913 \\
\hline Methanol & 22.5 & -0.09 & 175.2 & 512.6 & 29.8 & 30.6 & 33.10 & 0.9935 & 0.9821 \\
\hline Toluene & 28.5 & -0.10 & 178 & 591.8 & 38.8 & 41.1 & 40.00 & 0.9996 & 0.9938 \\
\hline Heptane & 20.1 & -0.10 & 182.55 & 540.1 & 30.6 & 31.3 & 31.15 & 0.9997 & 0.9904 \\
\hline Nonane & 22.8 & -0.09 & 219.45 & 594.6 & 31.4 & 30.7 & 29.42 & 0.9993 & 0.998 \\
\hline Octane & 21.6 & -0.09 & 216.65 & 568.8 & 29.8 & 28.6 & 28.47 & 0.9935 & 0.9821 \\
\hline
\end{tabular}

There is no meaningful relation between $\gamma_{\mathrm{m}}$ and $\mathrm{A}$, the coefficient of Pelofsky's equation. He and others tried to correlate A and surface tension but they were not successful. In fact, they attempted unsuccessfully to correlate the temperature where $\eta \rightarrow \infty$ (melting point) and $\gamma_{\mathrm{m}}$. But $\delta$, defined in eqn.3, approaches very well to $\gamma_{\mathrm{m}}$. For most liquids the difference are the order of experimental errors.

\section{Conclusion}

Surface tension and viscosity, highly important in process design and operation, are related to each other by the following new equation:

$$
\gamma=\delta \frac{T_{c}-T}{T_{c}-T_{m}}\left(1-\frac{\beta}{\eta}\right)
$$

The available literature data fit well in this developed equation. This equation satisfies both extreme conditions and $\delta$ is $\gamma_{\mathrm{m}}$, melting point of the liquid.

The result of this work shows that bulk transport property $(\eta)$ and surface property $(\gamma)$ are well interrelated.

As surface tension is a strong function of surfactants, emphasis on this fact that mixed components must not be surface active agents was addressed in this work, that is, any correlation between surface tension and viscosity can be valid in the absence of any surface active agents and capillarity.

\section{Acknowledgement}

Supports from the Department of Chemical Engineering, Quchan Branch, Islamic Azad University and the Department of Chemical Engineering, Isfahan
University of Technology are gratefully acknowledged.

\section{References}

1. A.W. Adamson, "Physical chemistry of surfaces," 1990: Wiley.

2. J.J. Bikerman, "Physical surfaces. 1970: Academic Press".

3. J.J. Jasper, "The Surface Tension of Pure Liquid Compounds,"Journal of Physical and Chemical Reference Data, 1972. 1(4): p. 841-1010.

4. R. Novakovic, "Surface and transport properties of Au-Sn liquid alloys," Surface Science, 2005. 599(1-3): p. 230-247.

5. M.C. Amiri and A.A. Dadkhah, "On reduction in the surface tension of water due to magnetic treatment. Colloids and Surfaces A: Physicochemical and Engineering Aspects," 2006. 278(1-3): p. 252-255.

6. A.H. Pelofsky, "Surface Tension-Viscosity Relation for Liquids," Journal of Chemical \& Engineering Data, 1966. 11(3): p. 394-397.

7. H. Schonhorn, "Surface Tension-Viscosity Relationship for Liquids," Journal of Chemical \& Engineering Data, 1967. 12(4): p. 524-525.

8. A.J. Queimada, "Generalized relation between surface tension and viscosity: a study on pure and mixed n-alkanes," Fluid Phase Equilibria, 2004. 222-223(0): p. 161-168.

9. K.S. Pedersen, A. Fredenslund and P. Thomassen, "Properties of Oils and Natural Gases, Contributions in Petroleum Engineering," Vol. 5. 1989, Houston: Gulf Publishing Company. 


\section{Chemical Engineering Research Bulletin 18(2015) 18-22}

10. A.J. Queimada, "Prediction of viscosities and surface tensions of fuels using a new corresponding states model," Fuel, 2006. 85(5-6): p. $874-877$.

\section{Available online at http://www.banglajol.info/index.php/CERB}

